\title{
Introduction to the Third Annual Lifelog Search Challenge (LSC'20)
}

\author{
Cathal Gurrin \\ Dublin City University \\ Ireland \\ Duc-Tien Dang-Nguyen \\ University of Bergen \\ Norway \\ Wolfgang Hürst \\ Utrecht University \\ Netherlands
}

\author{
Tu-Khiem Le \\ Dublin City University \\ Ireland \\ Björn Pór Jónsson \\ IT University of Copenhagen \\ Denmark \\ Minh-Triet Tran \\ University of Science \& VNU-HCM \\ Vietnam
}

\author{
Van-Tu Ninh \\ Dublin City University \\ Ireland \\ Jakub Lokoč \\ Charles University \\ Czech Republic
Klaus Schöffmann
Klagenfurt University
Austria

\begin{abstract}
The Lifelog Search Challenge (LSC) is an annual comparative benchmarking activity for comparing approaches to interactive retrieval from multi-modal lifelogs. LSC'20, the third such challenge, attracts fourteen participants with their interactive lifelog retrieval systems. These systems are comparatively evaluated in front of a live-audience at the LSC workshop at ACM ICMR'20 in Dublin, Ireland. This overview motivates the challenge, presents the dataset and system configuration used in the challenge, and briefly presents the participating teams.
\end{abstract}

\section{CCS CONCEPTS}

- Human-centered computing $\rightarrow$ Empirical studies in interaction design; • Information systems $\rightarrow$ Mobile information processing systems; Search interfaces.

\section{KEYWORDS}

Lifelog, interactive retrieval systems, benchmarking.

\section{ACM Reference Format:}

Cathal Gurrin, Tu-Khiem Le, Van-Tu Ninh, Duc-Tien Dang-Nguyen, Björn pór Jónsson, Jakub Lokoč, Wolfgang Hürst, Minh-Triet Tran, and Klaus Schöffmann. 2020. Introduction to the Third Annual Lifelog Search Challenge (LSC'20). In Proceedings of the 2020 International Conference on Multimedia Retrieval (ICMR '20), June 8-11, 2020, Dublin, Ireland. ACM, New York, NY, USA, 2 pages. https://doi.org/10.1145/3372278.3388043

\section{INTRODUCTION}

Lifelogging is the action of digitising one's everyday life activities with the aid of technology such as electronic devices and computer applications. It has been an active topic of research for almost two decades since the MyLifeBits project [5] introduced an early prototype digital lifelog in 2002. Since then, the proliferation of

Permission to make digital or hard copies of part or all of this work for personal or classroom use is granted without fee provided that copies are not made or distributed for profit or commercial advantage and that copies bear this notice and the full citation on the first page. Copyrights for third-party components of this work must be honored.

For all other uses, contact the owner/author(s).

ICMR '20, June 8-11, 2020, Dublin, Ireland

(C) 2020 Copyright held by the owner/author(s)

ACM ISBN 978-1-4503-7087-5/20/06.

https://doi.org/10.1145/3372278.3388043 low-cost sensing technologies and cheap storage have resulted in a massive increase in the volumes of personal lifelog data in existence. The Lifelog Search Challenge (LSC) comparative benchmarking workshop was founded in 2018 to answer a call for more robust and effective technologies to assist individuals in managing and locating content from these vast archives. The LSC workshop joins a number of related activities, such as the NTCIR-Lifelog tasks [8] (2016-2019) and the ImageCLEF Lifelog task [3] (2017-2019). Yet, it is different from these other challenges in that it focuses on evaluating approaches to real-time interactive retrieval from mediarich personal lifelogs [9], with a particular emphasis on evaluating the usability of the retrieval systems for novice users.

\section{LSC'20 DATASET}

LSC'20 uses a new multimodal dataset containing four months of lifelog data gathered by one active lifelogger. The dataset has been constructed by merging parts of the three NTCIR Lifelog datasets from 2016 [6], 2017 [7], and 2019 [8], with images from 2015, 2016 and 2018, respectively. It consists of an image dataset of 191,439 wearable camera images at $1024 \times 768$ resolution (38.5GB). They were captured using the OMG Autographer and Narrative Clip wearable cameras, typically at a rate of 1-3 per minute during waking hours. The images have been anonymised, which means that faces and most readable text have been blurred in a manual or semi-manual process. Accompanying the images is a collection of textual metadata, consisting of timestamps, physical activities, biometrics (all years except 2015), and locations of the individual for every minute. Finally, to reduce the barriers to participation, the images come with a list of visual concepts extracted from the nonredacted version of the image dataset, which includes bounding boxes for objects.

\section{LSC'20 BENCHMARKING CONFIGURATION}

The search challenge takes place during the ACM ICMR'20 conference in Dublin, Ireland. During the search challenge, each participating system is evaluated twice, once with its expert users and once with novice users. In order to evaluate each system, participating teams are required to return results to a host server for a number of novel retrieval topics under strict time-constraints. For each topic, a participating team has a user (expert or novice) 
use their system to find a relevant item and submit it to the host server. The host server maintains a countdown clock and actively evaluates submissions against a manually generated ground truth Throughout the competition, an overall score is maintained for each team, which is the summation of the scores of the topics that have been processed up until that point. For each topic, a score is given based on the time taken to find the relevant content and the number of incorrect items previously submitted by that team to the host server during that topic. Full details of the scoring equation can be found in a review of LSC'18 [9]. The overall winner of the challenge is the team with the highest score, across both expert and novice users, when all topics are processed.

\section{PARTICIPATING SYSTEMS}

For the 2020 edition of the Lifelog Search Challenge, fourteen participating teams take part, each of which has built an interactive lifelog retrieval engine for the live competition at the challenge. Following is a list of the challenge entries, along with a short description. Vitrivr [18], the winning system from 2019, provides an enhanced version of their video search engine. The VIRET Tool is an adapted video search tool that provides advanced options to construct temporal queries and browse results [11]. VRLE [4] is a refinement of the top performing system from LSC'18 that supports interactive querying within a fully-immersive VR environment. The Myscéal retrieval system is developed to explore query expansion and word embedding approaches to interactive retrieval [19] and introduces a novel concept-weighting methodology. LifeGraph [17], a knowledge graph for lifelogs, integrates external data to enrich the semantic content and organises it as a semantic graph. Exquisitor [10] is an interactive learning system designed for large-scale collections. Li et al. [14] introduce a multi-level interactive engine that indexes multi-level features and supports multiple query modes. LifeXplore is a video exploration and retrieval tool [13] combining feature map browsing, concept search and filtering, as well as hand-drawn sketching. Mai-Nguyen et al. [15] bring a interactive multimodal search engine with Query-to-Sample attention-based retrieval with the aim of enhancing query formulation using textto-image mapping. Che et al. [2] integrate relationship-mapping between objects and subjects in the lifelog images. LifeSeeker 2.0 is an enhancement to the 2019 tool of the same name [12], supporting interactive graph-based filtering. The FIRST system [20] is designed to be a flexible integration platform for easy use of new query workflows and visualization layouts. SOMHunter, the winning system from VBS 2020, is another adaptation of a video search engine combining temporal queries and a relevance feedback model with specific additions to support lifelog retrieval [16]. Finally, Voxento is a prototype voice-controlled lifelog retrieval engine [1].

\section{ACKNOWLEDGMENTS}

This publication has emanated from research supported in part by Science Foundation Ireland under grant numbers SFI/12/RC/2289 and SFI/13/RC/2106, Vingroup Innovation Foundation (VINIF) with project code VINIF.2019.DA19, Czech Science Foundation (GAČR) project 19-22071Y, and Vietnam Ireland Bilateral Education Exchange Programme (VIBE) 2019.

\section{REFERENCES}

[1] Ahmed Alateeq, Mark Roantree, and Cathal Gurrin. 2020. Voxento: A Prototype Voice-controlled Interactive Search Engine for Lifelogs. In Proceedings of the 2020 ACM Workshop on the Lifelog Search Challenge. June, 9 2020, Dublin, Ireland.

[2] Tai-Te Chu, Chia-Chun Chang, An-Zi Yen, Hen-Hsen Huang, and Hsin-Hsi Chen. 2020. Multimodal Retrieval through Relations between Subjects and Objects in Lifelog Images. In Proceedings of the 2020 ACM Workshop on the Lifelog Search Challenge. June, 9 2020, Dublin, Ireland.

[3] Duc-Tien Dang-Nguyen, Luca Piras, Michael Riegler, Liting Zhou, Matthias Lux, Minh-Triet Tran, Tu-Khiem Le, Van-Tu Ninh, and Cathal Gurrin. 2019. Overview of ImageCLEFlifelog 2019 : Solve My Life Puzzle and Lifelog Moment Retrieval. Working Notes of \{CLEF\} 2019 - Conference and Labs of the Evaluation Forum,Lugano, Switzerland. 09 Sept 2019 (2019), 9-12.

[4] Aaron Duane, Björn Pór Jónsson, and Cathal Gurrin. 2020. VRLE: Lifelog Interaction Prototype in Virtual Reality. In Proceedings of the 2020 ACM Workshop on the Lifelog Search Challenge. June, 9 2020, Dublin, Ireland.

[5] Jim Gemmell, Gordon Bell, Roger Lueder, Steven Drucker, and Curtis Wong. 2002. MyLifeBits: fulfilling the Memex vision. (December 2002), 235-238.

[6] Cathal Gurrin, Hideo Joho, Frank Hopfgartner, Liting Zhou, and Rami Albatal. 2016. NTCIR Lifelog: The First Test Collection for Lifelog Research. Proceedings of the 39th International ACM SIGIR conference on Research and Development in Information Retrieval - SIGIR '16 (2016), 705-708.

[7] Cathal Gurrin, Hideo Joho, Frank Hopfgartner, Liting Zhou, Rashmi Gupta, Rami Albatal, and Duc-Tien Dang-Nguyen. 2017. Overview of NTCIR-13 Lifelog-2 Task. Proceedings of NTCIR-13 (2017), 6-11.

[8] Cathal Gurrin, Hideo Joho, Frank Hopfgartner, Liting Zhou, Van-Tu Ninh, TuKhiem Le, Rami Albatal, Duc-Tien Dang-Nguyen, and Graham Healy. 2019. Advances in Lifelog Data Organisation and Retrieval at the NTCIR-14 Lifelog-3 Task. In NII Testbeds and Community for Information Access Research, Makoto P Kato, Yiqun Liu, Noriko Kando, and Charles L A Clarke (Eds.). Springer International Publishing, Cham, 16-28.

[9] Cathal Gurrin, Klaus Schoeffmann, Hideo Joho, Andreas Leibetseder, Liting Zhou, Aaron Duane, Dang Nguyen, Duc Tien, Michael Riegler, Luca Piras, et al. 2019. Comparing Approaches to Interactive Lifelog earch at the Lifelog Search Challenge (LSC2018). ITE Transactions on Media Technology and Applications 7, 2 (2019), 46-59.

[10] Omar Shahbaz Khan, Mathias Dybkjær Larsen, Liam Alex Sonto Poulsen, Björn pór Jónsson, Jan Zahálka, Stevan Rudinac, Dennis Koelma, and Marcel Worring. 2020. Exquisitor at the Lifelog Search Challenge. In Proceedings of the 2020 ACM Workshop on the Lifelog Search Challenge. June, 9 2020, Dublin, Ireland.

[11] Gregor Kovalcik, Vit Skrhak, Tomas Soucek, and Jakub Lokoc). 2020. VIRET Tool with Advanced Visual Browsing and Feedback. In Proceedings of the 2020 ACM Workshop on the Lifelog Search Challenge. June, 9 2020, Dublin, Ireland.

[12] Tu-Khiem Le, Van-Tu Ninh, Minh-Triet Tran, Thanh-An Nguyen, Hai-Dang Nguyen, Liting Zhou, Graham Healy, and Cathal Gurrin. 2020. LifeSeeker 2.0 : Interactive Lifelog Search Engine at LSC 2020. In Proceedings of the 2020 ACM Workshop on the Lifelog Search Challenge. June, 9 2020, Dublin, Ireland.

[13] Andreas Leibetseder and Klaus Schoeffmann. 2020. lifeXplore at the Lifelog Search Challenge 2020. In Proceedings of the 2020 ACM Workshop on the Lifelog Search Challenge. June, 9 2020, Dublin, Ireland.

[14] Jiayu Li, Min Zhang, Weizhi Ma, Yiqun Liu, and Shaoping Ma. 2020. A Multi-level Interactive Lifelog Search Engine with User Feedback. In Proceedings of the 2020 ACM Workshop on the Lifelog Search Challenge. June, 9 2020, Dublin, Ireland.

[15] Anh-Vu Mai-Nguyen, Van-Luon Tran, Trong-Dat Phan, Anh-Khoa Vo, Minh-Son Dao, and Koji Zettsu. 2020. BIDAL-HCMUS@LSC2020: An Interactive Multimodal Lifelog Retrieval with Query-to-Sample Attention-based Search Engine. In Proceedings of the 2020 ACM Workshop on the Lifelog Search Challenge (LSC '20). Association for Computing Machinery, June, 92020 , Dublin, Ireland.

[16] Frantisek Mejzlik, Patrik Veselý, Miroslav Kratochvíl, Tomáš Souček, and Jakub Lokoč. 2020. SOMHunter for Lifelog Search. In Proceedings of the 2020 ACM Workshop on the Lifelog Search Challenge. June, 9 2020, Dublin, Ireland.

[17] Luca Rossetto, Matthias Baumgartner, Narges Ashena, Florian Ruosch, Romana Pernischova, and Abraham Bernstein. 2020. LifeGraph: A Knowledge Graph for Lifelogs. In Proceedings of the 2020 ACM Workshop on the Lifelog Search Challenge. June, 9 2020, Dublin, Ireland.

[18] Loris Sauter, Silvan Heller, Mahnaz Amiri Parian, Ralph Gasser, and Heiko Schuldt. 2020. Interactive Lifelog Retrieval with vitrivr. In Proceedings of the 2020 ACM Workshop on the Lifelog Search Challenge. June, 9 2020, Dublin, Ireland.

[19] Ly Duyen Tran, Duy Nguyen, Binh Nguyen, Hyowon Lee, and Cathal Gurrin. 2020. Myscéal: An Experimental Interactive Lifelog Retrieval System for LSC'20. In Proceedings of the 2020 ACM Workshop on the Lifelog Search Challenge. June, 9 2020, Dublin, Ireland.

[20] Minh-Triet Tran, Thanh-An Nguyen, Quoc-Cuong Tran, Mai-Khiem Tran, Khanh Nguyen, Van-Tu Ninh, Tu-Khiem Le, Hai-Dang Nguyen, Trong-Le Do, Viet-Khoa Vo-Ho, and Cathal Gurrin. 2020. FIRST - Flexible Interactive Retrieval SysTem for Visual Lifelog Exploration at LSC 2020. In Proceedings of the 2020 ACM Workshop on the Lifelog Search Challenge. June, 9 2020, Dublin, Ireland. 